INTERNATIONAL JOURNAL OF SCIENTIFIC RESEARCH

\title{
RECURRENT CYSTOSACROMA PHYLLOIDES TUMOUR OF BREAST WITH LIPOSRCOMATOUS DIFFERENTIATION
}

\author{
General Surgery \\ Dr. R. \\ Jayaraghavan \\ Resident, Department of General Surgery, BJGMC, Pune.
}

\section{ABSTRACT}

Cysteosarcoma phylloides (CP) constitutes a rare form of breast neoplasia, which represents less than $1 \%$ of total breast neoplasias and only $2,3 \%$ of all fibro-epithelial breast tumors.It is termed "cystosarcoma phylloides" because of the extensions of the tumor mimicking the shape of leaves, intruding within the cystic cavities of the tumor. Phyllodes tumours of the breast are characterized by having both an epithelial as well as stromal component and these usually comprise almost $3 \%$ of all fibroepithelial tumours. They are exceptional in this aspect to convert into a stromal sarcoma of the breast after multiple recurrences. The stromal component of phyllodes tumor has the potential to undergo metaplasia to cartilage, bone, smooth muscle and striated muscle as well as their respective malignant neoplasms. The liposarcomatous differentiation of the stromal elements of phyllodes tumor is extremely rare. The recurrence rate is $16-28 \%$ after mastectomy or wide-wedge resection and $28-46 \%$ after simple enucleation of the tumor. The tumor metastasizes via the vascular route; about two thirds of metastases are to the lungs, fewer are to the bone, liver, and myocardium. Phylloides tumor occurs mostly in women in their fifth or sixth decade, and occasionally in women younger than 20 years old.

\section{KEYWORDS}

\section{Case Report}

A 48 year old female presented in with a lump in the left breast that had been gradually increasing for 2 months. Excision of the mass was done in another hospital 6 months back then it aggressively reoccurred again, there was no family history of the same illness. On presentation, the patient was alert, conscious and her vital signs were stable. On breast examination, $15 * 15 * 10 \mathrm{~cm}$ hard lobulated non tender mass occupied the entire left breast .Axillary lymph nodes not palpable. All other investigations were within normal limits. A Trucut biopsy showed a malignant phyllodes tumor. Computer tomography scanning for chest reveals $10 * 14 * 14 \mathrm{~cm}$ large well defined lobulated cystic lesion in left breast parenchyma in retroareolar region extending into all quadrants of breast predominantly in superior half. Computer tomography scanning for abdomen is normal.

The patient underwent total mastectomy without axillary dissection.

Cut section shows gelatinous slimy tumour mass which is well circumscribed and encapsulated. Microscopically myxoid stroma with arborizing capillarynetwork and atypical spindloid cells with occasional lipoblast like cells. Areas of cartilaginous and osteoid differentiation also seen. Epithelial element is not seen within the tumour suggestive of myxoid liposarcoma.
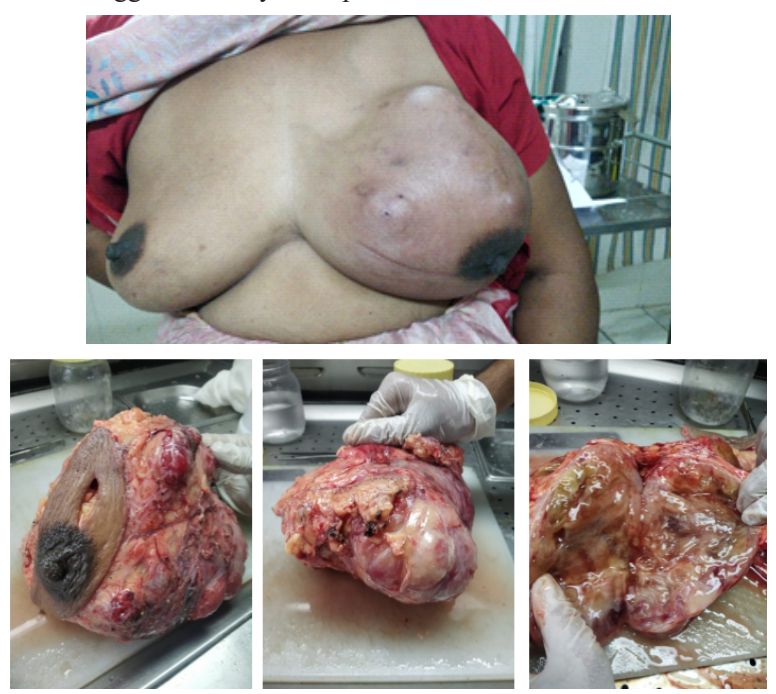

DISCUSSION

Tumors grow radially and compress the surrounding breast parenchyma, a false capsule is created, through which the tumor extends and grows into the rest of the healthy mammary tissue. The overlying skin is usually shiny and translucent enough to reveal underlying veins at its initial presentation. Ultimately, the tumor can cause an ulcer or open wound on the skin. These tumors represent a character of sizeable malignant sarcoma, taking a leaf-like appearance on gross examination and cystic spaces on histological examination In most cases, it mimics a benign breast condition such as fibroadenoma, making the diagnosis more challenging unless it grows to a large, ulcerative, hemorrhagic lesion. The tumor differentiates from other benign breast disorders by the increased mitotic activity, cellular atypia, and stromal proliferation. Although the malignant potential is very rare, lungs are the most common metastatic site, followed by the skeleton, heart and liver. The stromal element is more cellular than a fibroadenoma and consists of spindle cells with infrequent mitosis. Occassional cases may show admixture of adipose tissue foci. stromal elements in malignant phyllodes tumours usually exhibit a fibrosarcomatous differentiation. Heterologous differentiation such as chondrosarcoma, osteosarcoma, angiosarcoma, leiomyosarcoma, rhabdomyosarcoma and liposarcima are rarely encountered in malignant phyllodes tumours. Liposarcomatous differentiation is extremely rare and consist of well differentiated round cell, myxoid and pleomorphic liposarcomatous components. Liposarcomatous differentiation of the stromal component, however, does not appear to correlate with aggressive clinical behaviour. Phyllodes tumour with liposarcomatous differentiation requires complete surgical excision to prevent local tumour recurrence.Treatment of PT is surgical, regardless of subtype, ranging from wide local excision to mastectomy. Currently, there is a greater tendency towards conservative approaches, because PT is rarely multifocal. Recent guidelines recommend that PT $>3 \mathrm{~cm}$ be resected with free margins $\geq 1 \quad \mathrm{~cm}$, with no need for axillary staging; lumpectomy may be indicated for small tumors, because it allows preservation of mammary function. Some studies suggest that patients with benign and borderline PTs $>5-10 \mathrm{~cm}$ or with other risk factors for recurrence and metastasis, malignant PTs, more than three recurrences or positive resection margin, or age above 50 years, should be subjected to mastectomy . resection. Because our patient had progressive, borderline phylloides tumour $>10 \mathrm{~cm}$ simple mastectomy on the left was chosen.

\section{Conclusion}

Accurate preoperative pathological diagnosis allows correct surgical planning and avoidance of reoperation. The value of FNAC in the diagnosis of phyllodes tumor remains controversial, but core needle biopsy has high sensitivity value. Surgical management is the mainstay, whereas radiotherapy and chemotherapy are controversial and local recurrence in phyllodes tumors has been associated with inadequate local excision.

\section{References}

1. Garlet BB, Zogbi L, Lima JP, Favalli PPS, Krahe FD. Recurrent borderline phyllodes tumor of the breast submitted to mastectomy and immediate reconstruction: Case report. Int J Surg Case Rep. 2019;60:25-29. doi: 10.1016/j.ijscr.2019.05.032. Epub 2019 Jun 5. PMID: 31195364; PMCID: PMC6562175.

2. Hansen JR. Recurrent cystosarcoma phylloides. Calif Med. 1967 Jun;106(6):462-5. PMID: 4292573; PMCID: PMC1502678 
3. Stamatakos M, Tsaknaki S, Kontzoglou K, Gogas J, Kostakis A, Safioleas M. Phylloides tumor of the breast: a rare neoplasm, though not that innocent. Int Semin Surg Oncol. 2009 Feb 20;6:6. doi: 10.1186/1477-7800-6-6. PMID: 19232098; PMCID: PMC2649148.

4. Semiglazov VV, Shu V, Tabagua TT, Semiglazova TY. [Diagnosis and treatment of phylloides tumors of the breast]. Vopr Onkol. 2016;62(3):552-9. Russian. PMID: 30463117 . 